\title{
Berheim "a" wave: obstructed right ventricular inflow or atrial cross talk?
}

\author{
Michael Y Henein, Han B Xiao, Stephen J D Brecker, Derek G Gibson
}

\begin{abstract}
Objective-To study the possible mechanisms underlying the dominant " $a$ " wave in the jugular venous pulse seen in patients with left ventricular hypertrophy (Bernheim "a" wave).

Design-Prospective examination of the left ventricular transverse and longitudinal axes, transmitral and transtricuspid flows, and jugular venous pulse recordings.

Setting-Tertiary referral centre for cardiac disease.

Subjects-23 patients with left ventricular hypertrophy of various aetiologies and a dominant " $a$ " wave in the jugular venous pulse. Controls were 21 normal volunteers.
\end{abstract}

Results-Early diastolic filling of the right ventricle was normal. During right atrial systole the (mean(SD)) tricuspid ring motion was exaggerated $(1 \cdot 2(0 \cdot 4) v$ $0.8(0.2) \mathrm{cm}, p<0.001)$ and Doppler $A$ wave velocity slightly increased $(0 \cdot 3(0 \cdot 1)$ $v 0.2(0.08) \mathrm{m} / \mathrm{s}, \mathrm{p}<0.01)$, although the $E$ wave remained dominant. By contrast left ventricular isovolumic relaxation time was longer than normal $(70(20) v$ 55(10) ms, p $<0.001$ ) with wall motion incoordinate in the septal long axis, $15 \%(9.5 \%) v 6.6 \%(3 \%)$ total excursion occurring before mitral valve opening. During early filling the extent of long axis motion was decreased to $0.6(0.5) \mathrm{cm}$ from 1.1(0.2) cm, $(p<0.001)$ and $0.5(0 \cdot 2)$ cm from $0.9(0.2) \mathrm{cm},(p<0.0001)$ at the left and septal sites, and similarly its peak lengthening rate reduced to $5 \cdot 4(2 \cdot 5)$ $\mathrm{cm} / \mathrm{s}$ from $10(3) \mathrm{cm},(\mathrm{p}<0.001)$ and $4 \cdot 3(2 \cdot 2) \mathrm{cm} / \mathrm{s}$ from $8(2) \mathrm{cm}$, $(p<0.001)$. The atrial component of long axis lengthening was increased to $43 \%(18 \%)$ from $29 \%(6 \%) \quad(p<0.01)$ and $55 \%(15 \%)$ from $33 \%(8 \%)$ of the total excursion $(p<0.0001)$. Left ventricular E/A ratio was less than normal $(0 \cdot 9(0 \cdot 8) v 1 \cdot 4(0 \cdot 4)$, p < 0.05).

Conclusions-There is no evidence of obstruction or any other disturbance of early diastolic right ventricular inflow in Bernheim's syndrome. It is possible that the haemodynamically appropriate increase in left atrial activity is mirrored on the right side due to shared interatrial myocardial fibres. This could represent a form of atrial interaction.

(Br Heart f 1993;69:409-413)
An " $a$ " wave is commonly seen in the jugular venous pulse in patients with left ventricular hypertrophy, and is traditionally referred to as being part of the Bernheim syndrome. It has been ascribed to distortion of the right ventricular cavity by a bulging interventricular septum, ${ }^{1}$ or to abnormal diastolic function of the left ventricle affecting that of the right. ${ }^{2}$ Wood, while commenting on this association between left sided hypertrophy and a right sided "a" wave, pointed out that although necropsy evidence of obstruction to the right sided inflow was suggestive, definite proof for this mechanism was lacking. ${ }^{3}$ It therefore seemed of interest to reinvestigate the question with echocardiographic and Doppler techniques, as these approaches have proved useful in studying other disturbances of ventricular filling.

\section{Patients and methods}

PATIENTS

M mode echocardiograms, Doppler traces, and jugular venous pulses were recorded in 23 patients with a dominant " $a$ " wave in the venous pulse and left ventricular hypertrophy. We excluded patients with clinically apparent coronary artery disease, organic mitral valve disease, increased overall venous pressure, or more than mild aortic regurgitation causing left ventricular cavity enlargement. Six patients had left ventricular hypertrophy due to hypertrophic cardiomyopathy, 13 had aortic valve stenosis, two had undergone prosthetic aortic valve replacement, and two had systemic hypertension. All had a normal left ventricular cavity size. There were 15 men and eight women aged 21 to 82 years (mean (SD) 62 (18)). Twenty one additional healthy volunteers, 13 men and eight women of mean (SD) age 51 (11) years without clinical, electrocardiographic, or echocardiographic evidence of heart disease served as normal controls.

\section{METHODS}

$M$ mode and cross sectional echocardiograms were taken with a Hewelett Packard Sonos 1000 echocardiograph and a $2.5 \mathrm{MHz}$ phased array transducer as the patient lay in the partial left lateral position. Standard $M$ mode echocardiograms of the left ventricular minor axis were recorded just below the level of the mitral valve leaflets showing clear continuous echoes from both surfaces of the septum and posterior wall. 
Septal and posterior wall thicknesses were measured from their leading edges at the time of the onset of the $Q$ wave of the electrocardiogram (end diastole). The timing of aortic valve closure was taken as the onset of the first high frequency vibration of the aortic component of the second heart sound, and isovolumic relaxation as the time interval between this and the onset of separation of mitral valve leaflets.

Cross sectionally guided $M$ mode echocardiograms of the left and right ventricular long axes were then taken after longitudinal placement of an $M$ mode cursor from the apex through the atrioventricular ring at the three standard sites; left, septal (central fibrous body), and right, visualised on an apical four chamber cross sectional view. ${ }^{4}$ Minor and long axis echocardiograms were digitised. ${ }^{5}$

\section{DOPPLER}

Transmitral and transtricuspid forward flow velocities were recorded at cusp tip level with Hewlett Packard or Doptek equipment in pulsed mode ( 2.0 or $3.5 \mathrm{MHz}$ transducer).

\section{VENOUS PULSE}

The jugular venous pulse was recorded from the internal jugular vein on the right side of the neck with the patient lying supine in the optimal position, often less than 45 degrees, as overall venous pressure was not significantly raised in any patient. A Cambridge pulse transducer was used with a time constant of $4 \mathrm{~s}$.

All records were made photographically at a paper speed of $10 \mathrm{~cm} / \mathrm{s}$ with a Honeywell (Ecoline) strip chart recorder with simultaneous electrocardiogram (lead II) and phonocardiogram.

\section{MEASUREMENTS}

From the original traces, we measured left ventricular end diastolic and end systolic dimensions, septal thickness, and isovolumic relaxation time. Peak rate of increase of transverse dimension was derived by digitisation. On the pulsed Doppler traces of the transmitral and transtricuspid flow we measured peak $E$ and $A$ wave velocities and found the corresponding E/A ratio.

From the long axis traces (fig 2), we measured the overall amplitude of excursion and any change in dimension during isovolumic relaxation. Early diastolic lengthening was taken as the increase in length from the minimum value to that at the onset of diastasis; and atrial contraction was the further backward displacement of the atrioventricular ring toward the atrium after the $P$ wave. From the digitised traces, the peak rate of early diastolic lengthening was measured at each of the three sites of the long axis.

Jugular venous pulse traces were assessed with reference to the presence of " $x$ " or " $y$ " descents accompanying the " $a$ " waves. An " $x$ " descent was taken as a fall in venous pressure, of amplitude one quarter that of the " $a$ " wave that was complete before $\mathrm{P}_{2}$ (the pulmonary component of the second heart sound) on the phonocardiogram; a " $y$ " descent was one of similar amplitude occurring after $\mathbf{P}_{2}$.

\section{Results}

RIGHT VENTRICLE

Tables 1-4 show the results. Right ventricular filling pattern was effectively normal, showing a dominant $\mathrm{E}$ wave on the transtricuspid Doppler trace. The amplitude of the " $a$ " wave was slightly although consistently larger than normal $(p<0.01)$. Early diastolic excursion of the ring was normal in both absolute and relative terms, as was peak lengthening rate. The excursion during atrial systole was consistently but modestly increased $(p<$ $0.001)$. The jugular venous pulse showed a dominant "a" wave in all patients, by definition. Additional small " $x$ " descents were present in 22 patients and a " $y$ " descent in one. (fig 1)

Table 1 Minor axis measurements (mean (SD))

\begin{tabular}{llll}
\hline Variable & Normal & Bernheim & $p$ Value \\
\hline Minor axis excursion $(\mathrm{cm})$ & $1 \cdot 7(0 \cdot 3)$ & $1 \cdot 9(0 \cdot 6)$ & NS \\
End diastolic diameter $(\mathrm{cm})$ & $4 \cdot 9(0 \cdot 5)$ & $4 \cdot 5(1)$ & NS \\
End systolic diameter $(\mathrm{cm})$ & $3 \cdot 3(0 \cdot 5)$ & $3 \cdot 0(1)$ & NS \\
Shortening fraction & $0 \cdot 3(0 \cdot 1)$ & $0 \cdot 3(0 \cdot 1)$ & NS \\
Septal thickness $(\mathrm{cm})$ & $1 \cdot 0(0 \cdot 1)$ & $1 \cdot 5(0 \cdot 4)$ & $\star$ \\
Posterior wall thickness $(\mathrm{cm})$ & $1 \cdot 0(0 \cdot 1)$ & $1 \cdot 3(0 \cdot 3)$ & $\star$ \\
Peak thinning rate $(\mathrm{cm} / \mathrm{s})$ & $11(2 \cdot 7)$ & $8 \cdot 2(3.6)$ & $<0.01$ \\
\hline
\end{tabular}

$\star$ Significantly different by definition.

Table 2 Long axis measurements (mean (SD))

\begin{tabular}{|c|c|c|c|}
\hline Variable & Normal & Bernheim & p Value \\
\hline cursion $(\mathrm{cm}) \mathrm{L}$ & $1 \cdot 5(0.3)$ & $1 \cdot 2(0 \cdot 4)$ & \\
\hline min & 1.4 & & \\
\hline Total excursion $(\mathrm{cm}) \mathbf{R}$ & $2 \cdot 5(0 \cdot 4)$ & $2 \cdot 6(0 \cdot 7)$ & $N$ \\
\hline y diastole $(\mathrm{cm}) \mathrm{L}$ & $1 \cdot 1$ & & \\
\hline lastole $(\mathrm{cm}) \mathrm{S}$ & $0.9(0.2)$ & 0.5 & $<0.001$ \\
\hline astole $(\mathrm{cm}) \mathbf{R}$ & $1 \cdot 6(0 \cdot 3)$ & 1.4 & $\mathbf{N}$ \\
\hline A wave $(\mathrm{cm}) \mathrm{L}$ & $0.4(0.1)$ & 0.5( & \\
\hline$(\mathrm{cm}) \mathrm{S}$ & $0.5(0 \cdot 2)$ & 0.6 & \\
\hline lal A wave (cm) $R$ & $0 \cdot 8(0 \cdot 2)$ & $1 \cdot 2(0.4)$ & $<0.0$ \\
\hline r & & & \\
\hline ial-total excursion(\%) S & 33( & 55 & $<$ \\
\hline trial-total excursion(\%) $\mathbf{R}$ & 33( & $46(13)$ & $<0.001$ \\
\hline$(\mathrm{cm} / \mathrm{s}) \mathrm{L}$ & $10(3$ & $5 \cdot 4(2 \cdot 5)$ & \\
\hline eak lengthening rate $(\mathrm{cm} / \mathrm{s}) \overline{\mathrm{S}}$ & $8(2)$ & $4 \cdot 3(2 \cdot 2)$ & $<0.0$ \\
\hline eak lengthening & $10(2 \cdot 6)$ & $10 \cdot 3(4)$ & \\
\hline
\end{tabular}

$\mathrm{L}$, left; $\mathrm{S}$, septal; $\mathrm{R}$, right sites of atrioventricular ring motion.

Table 3 Isovolumic relaxation measurements (mean (SD)) Variable Normal Bernheim p Value

A $_{2}$-mitral valve opening (ms) $55(10) 70(20)<0.001$

Dimension changes during IVR(\%):

Minor axis

Long axis $L$

Long axis $S$

Long axis $\mathbf{R}$

8.6 (5) 12 (9) NS $10(7.5) 12(11)$ NS $6.6(3) 15(9.5)<0.01$ $11(9) \quad 15(12)$ NS

IVR, Isovolumic relaxation; other abbreviations as for table 2 .

Table 4 Pulsed Doppler measurements (mean (SD))

\begin{tabular}{|c|c|c|c|}
\hline \multirow{2}{*}{$\frac{\text { Table } 4}{\text { Variable }}$} & \multirow[b]{2}{*}{ Normal } & \multirow[b]{2}{*}{ Bernheim } & \multirow[b]{2}{*}{ p Value } \\
\hline & & & \\
\hline \multirow{4}{*}{$\begin{array}{l}\text { Mitral E wave velocity }(\mathrm{m} / \mathrm{s}) \\
\text { Mitral A wave velocity }(\mathrm{m} / \mathrm{s}) \\
\text { Mitral E/A ratio } \\
\text { Tricuspid E wave velocity } \\
(\mathrm{m} / \mathrm{s})\end{array}$} & $0.7(0.1)$ & $0.55(0.3)$ & $<0.05$ \\
\hline & $0.5(0.1)$ & $0.7(0.4)$ & $<0.05$ \\
\hline & $1.4(0.4)$ & $0.9(0.8)$ & $<0.05$ \\
\hline & $0.4(0.15)$ & $0.5(0 \cdot 2)$ & NS \\
\hline $\begin{array}{l}\text { Tricuspid A wave velocity } \\
(\mathrm{m} / \mathrm{s})\end{array}$ & $0.2(0.08)$ & $0.3(0.1)$ & $<0.01$ \\
\hline Tricuspid E/A ratio & $1.9(0.4)$ & $1.6(0.5)$ & $<0.05$ \\
\hline
\end{tabular}

$\mathrm{E}$, early diastolic; $\mathrm{A}$, late diastolic. 
Figure 1 fugular venous pulse (FVP) recording with simultaneous

electrocardiogram (ECG)

and phonocordiogram

(PCG) from a patient with left ventricular hypertrophy. Note the dominant " $a$ " wave and " $x$ " descent.

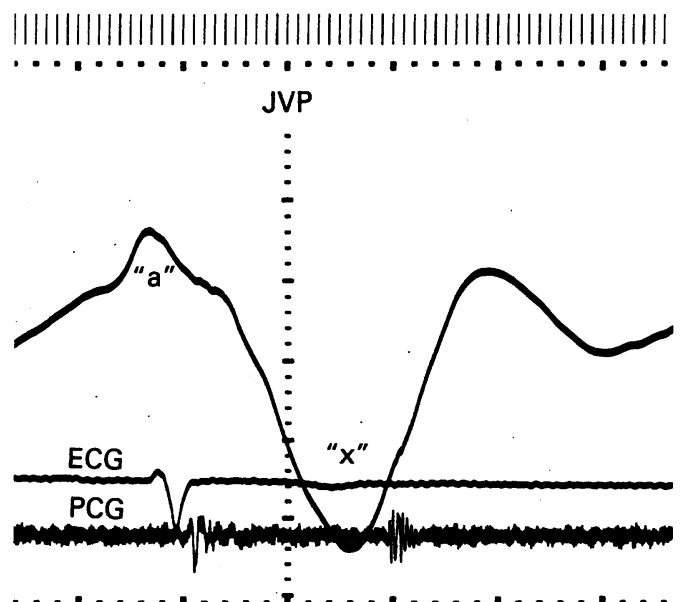

||||||||||||||||||||||||||||||||||||||||||||||||||||||||||||||||||

\section{LEFT VENTRICLE}

On $M$ mode, left ventricular transverse dimensions and shortening fraction were all normal. Isovolumic relaxation time, however, was prolonged $(p<0.001)$ and the peak rate of increase of transverse dimension was reduced $(p<0.01)$. Long axis motion was also abnormal. Its overall excursion was reduced due to a significant reduction in the early diastolic component, affecting both free wall and septum (both $\mathrm{p}<0.001$ ). This was accompanied by a decrease in peak rate of early diastolic lengthening at both sites $(p<$ $0.001)$. The amplitude of the early diastolic component included the lengthening that occurred during isovolumic relaxation. This was significantly increased at the septum ( $p<$ 0.01 ); so that the lengthening that occurred during filling itself - that is, after mitral valve opening - was even further reduced here in the patients with left ventricular hypertrophy (fig 2).

On the transmitral flow velocity trace, $\mathrm{E}$ wave velocities were reduced and $A$ wave velocities significantly increased (both $\mathrm{p}<0.05$ ) with respect to normal, so that the $\mathrm{E} / \mathrm{A}$ ratio was correspondingly low $(\mathrm{p}<0.05$, fig 3)

\section{Discussion}

Our patients fulfilled the accepted criteria for a Bernheim " $a$ " wave as they appear in recent publications. ${ }^{12}$ All had left ventricular hypertrophy with a dominant presystolic wave in the jugular venous pulse. We found no evidence of separate right sided disease, which might have provided an alternative explanation, and although we did not undertake invasive studies, a normal or prolonged left ventricular isovolumic relaxation time strongly suggests that mean left atrial pressure was normal. The presystolic wave in the venous pulse corresponded with the atrial component of transtricuspid flow, which, in all but one patient, could be clearly dissociated from the early diastolic component or $\mathrm{E}$ wave. Finally, the amplitude of tricuspid ring motion and transtricuspid flow velocities, although normal in early diastole, were both increased above normal during atrial systole,
Figure 2 (A) Long axis $M$ mode recordings from a normal person, taken at the left $(L)$, septal $(S)$, and right $(R)$ sites of atrioventricular ring motion showing the normal relations between early $(E)$, late $(A)$, and total (T) diastolic excursions. (B) Long axis $M$ mode recording from a patient with aortic stenosis and left ventricular hypertrophy. Note the decrease in early diastolic excursion and rate of lengthening, and also the relative increase in $A$ at all three sites. Both recordings are made with simultaneous

electrocardiogram (ECG) and phonocardiogram (PCG). Vertical line corresponds to the timing of aortic closure (A2).

Vertical scale is in $\mathrm{cm}$.
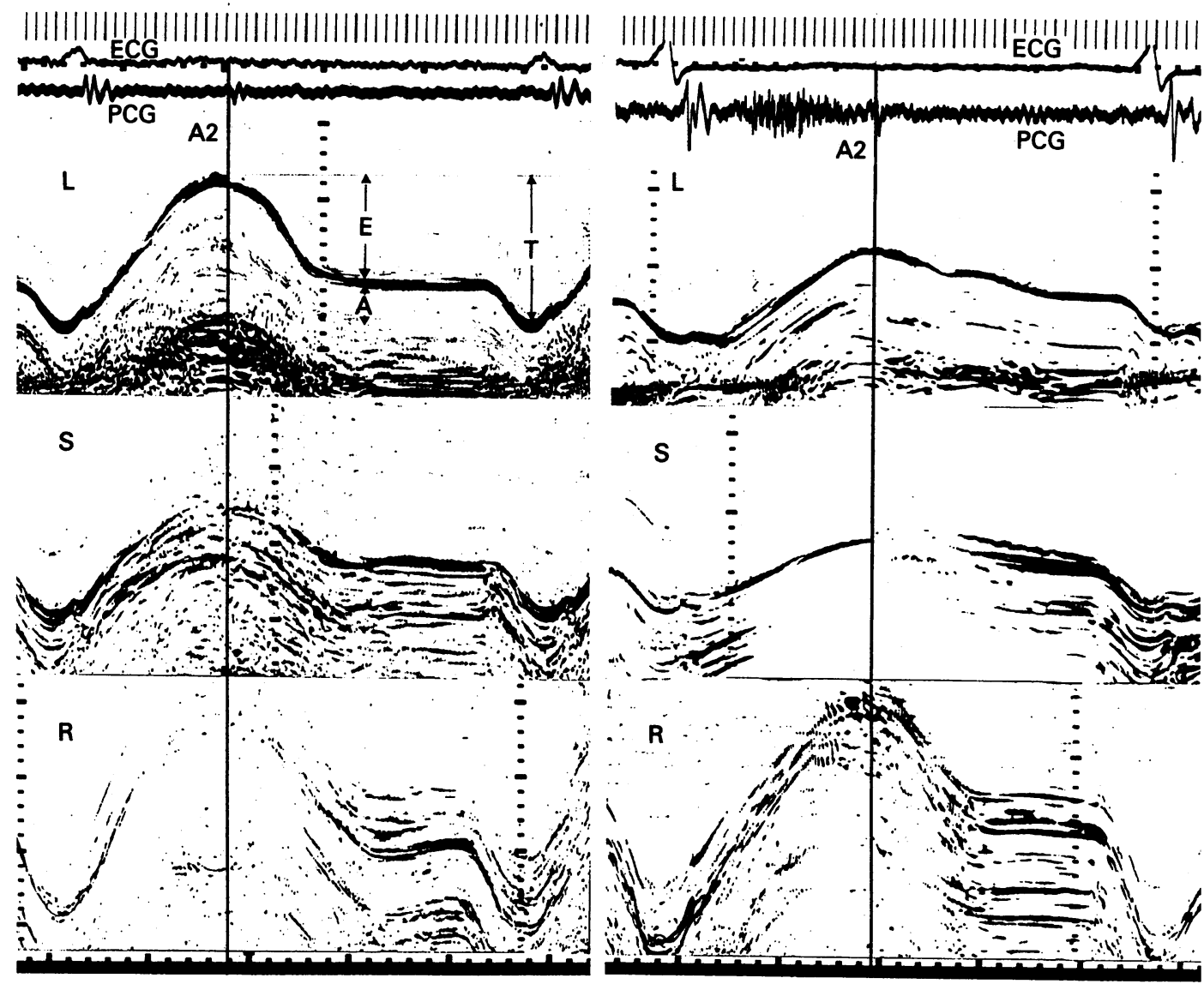
Figure 3 (A) Pulsed wave Doppler transmitral flow velocity trace from a patient with aortic stenosis and left ventricular hypertrophy. Note the dominant late diastolic $A$ wave and small $E / A$ ratio. (B) Pulsed wave Doppler trace of the tricuspid flow velocities from the same patient. Note the normal $E$ wave and $E / A$ ratio. Abbreviations as for fig 2.
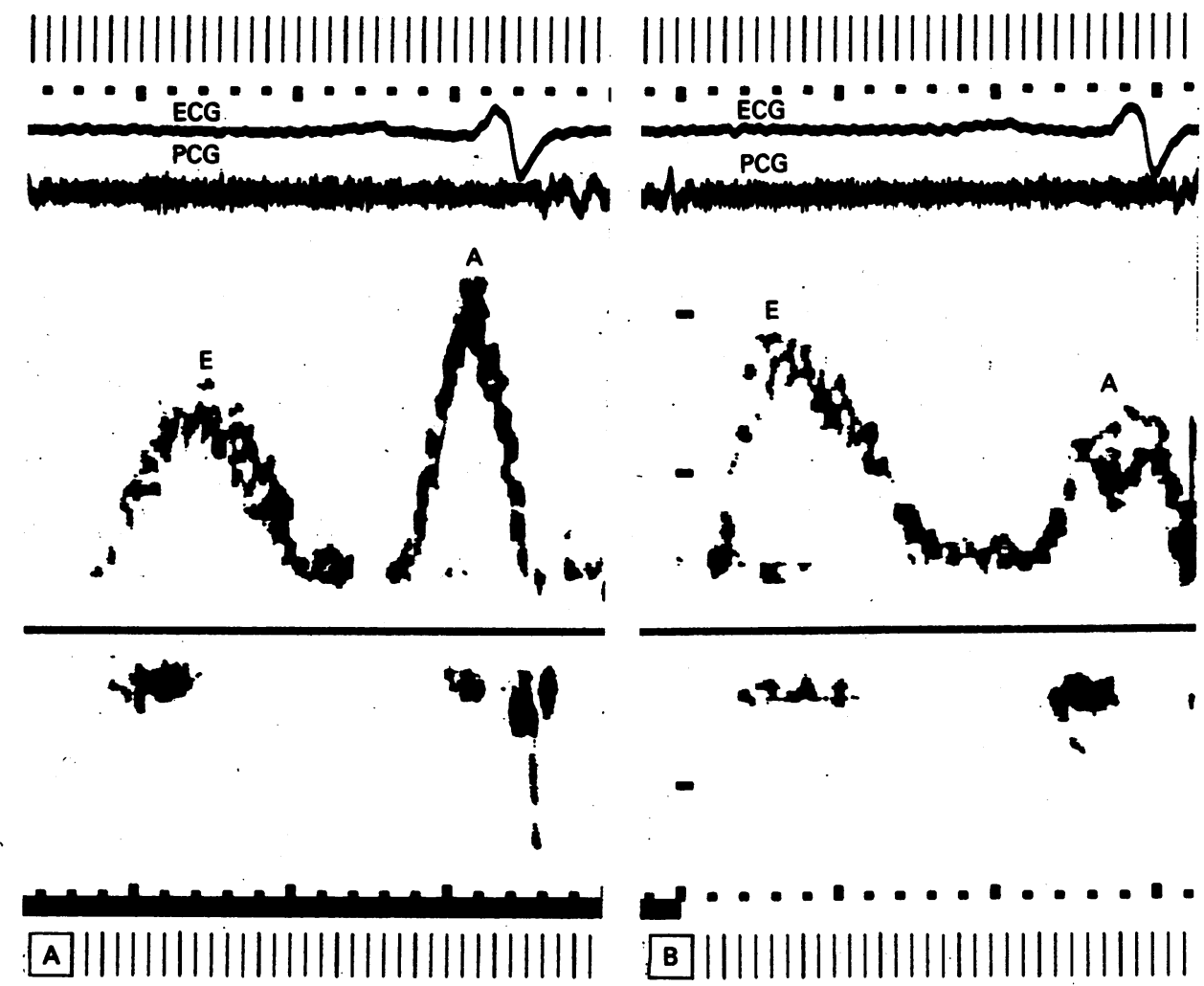

providing further evidence for abnormal mechanical activity of the right atrium. We conclude, therefore, that a true " $a$ " wave can occur in the jugular venous pulse of patients with left ventricular hypertrophy. Its mechanism differs fundamentally from the presystolic wave seen in those with dilated cardiomyopathy ${ }^{6}$ or severe pulmonary hypertension, ${ }^{7}$ where a very short filling time allows only a single summation flow pulse across the valve.

The name of Bernheim is associated with the syndrome under study because he suggested that left ventricular disease might directly cause right ventricular stenosis, thereby causing "l'asystole veineuse".8 Bernheim himself made no objective observations of the venous pulse, though he noted that the neck veins were engorged in his patients. The idea of venous asystole is no longer current, but a few years before Bernheim, Mackenzie had ascribed the " $v$ " wave of severe ventricular disease to right atrial paralysis. 9 The exact nature of the "stenose" of the right heart produced by bulging of interventricular septum has never been clearly delineated. On general grounds its effect on ventricular filling might manifest itself as physical obstruction of the right ventricular inflow tract, interference with the mechanisms underlying rapid right ventricular filling, or as increased right ventricular passive stiffness. We found no evidence of any of these. Although overall venous pressure was not raised, early diastolic $E$ wave velocities were normal on transtricuspid Doppler. This finding excluded both physical obstruction to the inflow and significant impairment of early diastolic filling mechanisms, whereas a normal venous pressure until the end of diastasis would seem to exclude any clinically important loss of ventricular compliance. In our patients, therefore, we found the rather unexpected combination of enhanced right atrial activity with normal early diastolic filling.

The unusual state of affairs on the right side of the heart is highlighted by comparing it with that on the left. Although left ventricular cavity size and shortening fraction were both normal, isovolumic relaxation time was prolonged and wall motion before mitral valve opening was incoordinate. We have previously shown that both of these abnormalities independently reduce the amplitude of the $E$ wave on the transmitral Doppler trace. ${ }^{10}$ At the same time, the absolute extent of early diastolic lengthening of the long axis fell as did the increase in the peak rate of transverse and long axes. As a result of these disturbances to early diastolic filling, A wave flow velocities were increased as was the relative increase in the long axis during atrial sysright side of the heart, therefore, there was clear evidence on the left that isovolumic relaxation and early diastolic filling were both abnormal, with a corresponding increase in ventricular volume during atrial systole.

The setting in which increased atrial activity occurred thus differed considerably on the two sides of the heart. On the left, it seemed homeostatic, the increased atrial contribution compensating for the reduced early diastolic flow. This allowed stroke volume to be maintained at normal filling pressure by mechanisms that are well understood. Increased right atrial activity cannot be so easily explained. Early diastolic flow velocity was normal. The atrial component of the transtricuspid flow was small and the increased pressure "a" wave would, if anything, reduce tole. By obvious contrast to events on the 
venous return. It must thus be asked whether the increased right atrial activity was pathological rather than homeostatic. The theoretical possibility of an inappropriate increase in the force of atrial contraction has been little explored. The transfer function between the force of atrial contraction and the nature and extent of ventricular disease is poorly understood. It might have been disturbed in our patients, in some unspecified way, perhaps by the septal hypertrophy. An alternative explanation might depend on the muscular anatomy of the atria being very simple compared with that of the ventricles. Even right or left ventricular hypertrophy cannot exist in isolation due to the common interventricular septum. As well as muscle bundles confined to one or other atrium, additional musculature is described as running continuously around the whole atrial cavity, unrelated to the interatrial septum. ${ }^{11}$ We suggest, therefore, that "cross talk" 12 between the two atria might occur, so that increased mechanical activity resulting directly from ventricular disease might not be confined to the atrium involved. Cross talk is used in communication theory to imply unwanted spread of information from the channel carrying it to another, where it is irrelevant or even corrupting. Here in the present paper, we mean to suggest that the communication channel "overheard" is that between left ventricle and left atrium: information arising from some aspect of diastolic left ventricular disease is transmitted to the left atrium causing its force of contraction to increase. This information flow spreads to the right atrium making its behaviour inappropriate. If this explanation is correct, it might also apply in other diseases, and to either side of the heart.

Our results also raise the question of how the phenomenon should be named. There was no evidence of the mechanical obstruction to right ventricular filling invoked by Bernheim, ${ }^{8}$ neither is a modest " $a$ " wave superimposed on an otherwise normal venous pressure in any way similar to the dilated neck veins, fluid retention, and hepatic engorgement that he described. We would resist any change. Eponyms, even when inaccurate or fanciful, have proved an effective means of identifying clinical syndromes that reflect unexpected associations, at a time when their underlying mechanisms are unclear. We suggest that rather than trying to read into Bernheim's original papers ideas that he never clearly entertained, the claims of tradition should be respected. More important, however, the mechanisms underlying these "a" waves should be investigated further. In doing so, understanding of interatrial and atrioventricular interactions will undoubtedly be increased.

Presented at the 65 th scientific session of the American Heart Association.

1 Hall R. Aortic stenosis. In:Julian DG, Camm AJ, Fox $\mathrm{KM}$, et al, eds. Diseases of the heart. London: Bailliere Tindall, 1989:705-30.

2 Shapiro LM. Left ventricular hypertrophy. In Julian DG, Camm AJ, Fox KM, et al, eds. Diseases of the heart. London: Bailliere Tindall, 1989:1414-25.

3 Wood P. Hypertensive heart disease. In: Wood P, ed Diseases of the heart and circulation. London: Eyre and Diseases of the heart and circulat
Spottiswoode, 1953:416-43.

4 Jones CJH, Raposo L, Gibson DG. Functional importance of the long axis dynamics of the human left ventritance of the long axis dynamics

5 Gibson DG, Brown D. Measurement of instantaneous left ventricular dimension and filling rate in man, using echocardiography. Br Heart $\mathcal{f}$ 1973;35:1 141-9.

6 Lee CH, Xiao HB, Gibson DG. Jugular venous "a" wave in dilated cardiomyopathy: sign of abbreviated right ventricular filling time. Br Heart 7 1991;65:342-5.

7 Stojnic BB, Brecker SJD, Xiao HB, Gibson DG. Jugular venous " $\mathrm{a}$ " in pulmonary hypertension: new insights from a Doppler echocardiographic study. $\mathrm{Br}$ Heart $\mathcal{f}$ 1992;68:187-91.

8 Bernheim HM. De l'asystolie veineuse dans l'hypertrophie du coeur gauche par stenose concomitante du ventricule droit. Revue de Medecine 1910;30:785-801.

9 Mackenzie J. Paralysis of the auricle and the ventricula form of the venous pulse. In: The study of the pulse. form of the venous pulse. In: The

10 Brecker SJD, Lee CH, Gibson DG. Relation of left ventricular isovolumic relaxation time and incoordination to transmitral Doppler filling patterns. $\mathrm{Br}$ Heart $\mathcal{f}$ 1992;68:567-73.

11 Williams PL, Warwick R. Myocardial architecture. In Gray's anatomy. 36th ed. Edinburgh: Churchill Livingstone 1980:657-9.

12 Amos SW. Dictionary of electronics, London: Butterworth 1981:69. 